\title{
C9orf100, a new member of the Dbl-family guanine nucleotide exchange factors, promotes cell proliferation and migration in hepatocellular carcinoma
}

\author{
HAIXIAO WANG ${ }^{1 *}$, YANDONG $\mathrm{LI}^{2,3 *}$, YUPING WANG ${ }^{2-4^{*}}$, ZE-GUANG HAN ${ }^{2,3}$ and BING CAI ${ }^{1}$ \\ ${ }^{1}$ Department of Hepatobiliary Surgery, Wuxi People's Hospital of Nanjing Medical University, Jiangsu; \\ ${ }^{2}$ Chinese National Human Genome Center, Rui-Jin Hospital, Shanghai Jiaotong University School of Medicine; \\ ${ }^{3}$ Shanghai-MOST Key Laboratory for Disease and Health Genomics, Chinese National Human Genome \\ Center at Shanghai, Shanghai; ${ }^{4}$ Department of Medical Biochemistry and Molecular Biology, \\ School of Basic Medical Sciences, Lanzhou University, Gansu, P.R. China
}

Received September 26, 2011; Accepted January 10, 2012

DOI: $10.3892 / \mathrm{mmr} .2012 .783$

\begin{abstract}
Dbl-family guanine nucleotide exchange factors (GEFs) are important activators of Rho GTPases, which are significantly associated with tumorigenesis and metastasis. The catalytic ability of the Dbl-family GEFs to activate Rho GTPases depends on their Dbl-homology domain followed by a pleckstrin-homology domain. In the present study, we showed that C9orf100, a new member of the Dbl-family GEFs with a minimal catalytic unit, may contribute to hepatocellular carcinoma (HCC). Quantitative real-time PCR results demonstrated that C9orf100 was highly and widely upregulated in 42/44 (95.5\%, >2-fold) HCC specimens compared with adjacent non-cancerous livers, and this upregulation was correlated with intrahepatic metastasis and $\alpha$-fetoprotein levels of HCC. Furthermore, the ectopic expression of C9orf100 promoted cell proliferation and colony formation in Huh-7 and YY-8103 cells, whereas silencing of C9orf100 resulted in the suppression of cell growth in MHCC-97H and PLC/PRF/5 cells. Flow cytometry confirmed this effect on MHCC-97H cell growth and indicated that $\mathrm{C} 9$ orf100 may function in the $\mathrm{G}_{2} / \mathrm{M}$ phase. In addition, we showed that C9orf100 is involved in the positive regulation of HCC cell migration by a transwell chamber
\end{abstract}

Correspondence to: Dr Bing Cai, Department of Hepatobiliary Surgery, Wuxi People's Hospital of Nanjing Medical University, 299 Qingyang Road, Wuxi, Jiangsu 214023, P.R. China E-mail: caibing@wuxiph.com

Dr Ze-Guang Han, Shanghai-MOST Key Laboratory for Disease and Health Genomics, Chinese National Human Genome Center at Shanghai, 351 Guo Shou-Jing Road, Shanghai 201203, P.R. China E-mail: hanzg@chgc.sh.cn

*Contributed equally

Key words: hepatocellular carcinoma, C9orf100, guanine nucleotide exchange factors, cell proliferation, cell migration analysis. Our findings suggest that $\mathrm{C} 9$ orf100 plays a potential oncogenic role in the development and metastasis of HCC.

\section{Introduction}

Hepatocellular carcinoma (HCC) is one of the most common and lethal malignancies worldwide, particularly in Asia and Africa. The main predisposing factors for its development include chronic infection with the hepatitis $\mathrm{B}$ and $\mathrm{C}$ virus, alcohol abuse and aflatoxin intake $(1,2)$. Cirrhosis induced by a virus or alcohol poses the highest risk, as $80 \%$ of HCCs develop in cirrhotic livers (3). The primary treatments of HCC are surgical resection, liver transplantation, percutaneous ablation and conventional chemotherapy; however, prognosis of advanced HCC remains poor (4). Tumor recurrence and metastasis in HCC treatment are still the main problems. As with many other tumors, the development and progression of HCC is a complex multistep process and the molecular mechanisms involved in this process have yet to be fully clarified. Ongoing investigations to identify new and effective molecular targets controlling $\mathrm{HCC}$ progression are crucial for improving prognosis and treatment strategies.

Activation of oncogenes and inactivation of tumor suppressor genes are frequently associated with carcinogenesis and progression. Identifying the genes that are differentially expressed in tumor from paratumor tissue is currently a topic of much interest. In recent years, with various transcriptome approaches, numerous upregulated oncogenes or downregulated tumor suppressor genes in $\mathrm{HCC}$ have been reported, such as IGF2, FAT10, SCARA5, DLK1 and p53 (5-9).

The Rho family of small GTPases are key regulators of a variety of cellular events: cell cycle progression, cytoskeletal reorganization, membrane trafficking, cell migration and invasion $(10,11)$. Previous studies have suggested that aberrant regulation of Rho family GTPases contributes to carcinogenesis and migration (12). Rho GTPases undergo a cycle between an active GTP-bound and inactive GDP-bound state, which is regulated mainly by the Dbl-family (diffuse B-cell lymphoma family) guanine nucleotide exchange factors 
(GEFs). Dbl-family GEFs are defined by the presence of a Dbl homology domain (DH domain) and this domain is almost invariantly followed by a pleckstrin homology domain (PH domain). The DH domain is responsible for catalyzing the exchange of GDP for GTP within Rho GTPases (13), while the $\mathrm{PH}$ domain acts as an anchor for targeting membrane (14). Previous studies have suggested that the PH domain is also involved in the protein-protein interactions (15). In the human genome, a total of 69 Dbl-family GEFs were found, all of which share a core catalytic unit consisting of the $\mathrm{DH}$ domain and an adjacent $\mathrm{PH}$ domain (16). In addition to the DH/PH module, most of the Dbl-family GEFs contain diverse structural domains such as serine/threonine kinase, RBD, PDZ, RGS, REM, RasGEF, Spectrin, CH, SH2 or SH3 (17). These domains are likely to integrate the upstream and downstream signals associated with GEFs. Several Dbl-family GEFs with additional functional domains, such as Dbl, Ect2, Vav3, were reported to be involved in tumor development and metastasis $(18,19)$. However, little is known in tumor about the Dbl-family GEFs that have no additional functional domains except for the $\mathrm{DH}$ and $\mathrm{PH}$ domains.

In this study, we identified a new member of the Dbl-family GEFs, C9orf100, which only shares a minimal catalytic unit compared with other members, and may contribute to the tumorigenesis and progression of HCC. Quantitative real-time PCR results showed C9orf100 was significantly upregulated in HCC tissues compared with related adjacent non-cancerous tissues, and this upregulation of C9orf100 was correlated, to some extent, with clinicopathological features of HCC. Further cell biological evidence indicated that C9orf100 promoted proliferation and migration of HCC cells in vitro. Thus, we propose that $C 9$ orf100 is a potential candidate oncogene in HCC.

\section{Materials and methods}

Tissue specimens and cell culture. All 44 specimens were harvested from patients suffering from HCC, who underwent resection of the primary tumor at the department of Wuxi People's Hospital. Informed consent was obtained from all patients and the use of the tissue material for research was approved by the Ethics Committee of the Wuxi People's Hospital and the Chinese National Human Genome Centre in Shanghai. The histopathological diagnosis was based on the criteria of the World Health Organization. Serum $\alpha$-fetoprotein (AFP) $\geq 10 \mathrm{ng} / \mathrm{ml}$ by chemoluminescence was diagnosed as positive. The paired paratumor liver samples were from the same patients and $2 \mathrm{~cm}$ away from the edge of tumor regions. Nine of the HCC patients had one tumor and 35 had multiple tumors. In addition, 37 patients were diagnosed with liver cirrhosis and 7 were not. HCC cell lines were obtained from our laboratory stocks. Cells were grown in Dulbecco's modified Eagle's medium (DMEM) supplemented with $10 \%$ fetal bovine serum (FBS; Gibco) and antibiotics (50 U/ml penicillin and $50 \mu \mathrm{g} / \mathrm{ml}$ streptomycin; Gibco) at $37^{\circ} \mathrm{C}$ in a $5 \% \mathrm{CO}_{2-}$ humidified incubator.

Quantitative real-time PCR. Total RNA was extracted from the tissue samples or the cultured cell lines using TRIzol reagent (Invitrogen) and was reverse transcribed into cDNA using the M-MLV reverse transcriptase kit (Promega, WI, USA). Quantitative real-time PCR was performed with the Thermal Cycler Dice Real-Time System (Takara) and SYBR-Green I reagent (Takara). The mRNA level of C9orf100 in each sample or HCC cells was normalized by $\beta$-actin prior to comparative analysis. The primer sets used were: $C 9$ orf100, forward: 5'-CAATTTGCTGCCAACTCAGA-3', reverse: 5'-GGACTCTCTGGGCAGTCTCA-3'; $\beta$-actin, forward: 5'-AGAGCCTCGCCTTTGCCGATCC-3', reverse: 5'-CTGGG CCTCGTCGCCCACATA-3'. Reactions were performed independently at least three times in triplicate.

Construction of recombinant plasmid. The full-length cDNA of C9orf100 (GeneBank accession no. NM_032818) was amplified from the human liver cDNA library and cloned into the pcDNA3.1 expression vector (Invitrogen) to generate Flagtagged pcDNA3.1-C9orf100 expression plasmid. Accurate reading frame insertion was verified by DNA sequencing.

RNA interference. To suppress C9orf100 expression in HCC cells, C9orf100-specific small interference RNAs (siRNAs) were chemically synthesized (GenePharma). The siRNA sequences used were: si-693 (sense: 5'-UCCAUACUAU UGGUCAGAAACAGdAdA-3' and antisense: 5'-UUCUGU UUCUGACCAAUAGUAUGdGdA-3'); si-1023 (sense: 5'-UCCCUCAUGAGAAGCUACUGCUU dAdT-3' and antisense: 5'-AUAAGCAGUAGCUUCUCAUGAG GdGdA-3'). The irrelevant nucleotides not targeting any annotated human genes were used as negative control: si-NC (sense: 5'-UUCUCC GAACGUGUCACGUdTdT-3' and antisense: 5'-ACGUGA CACGUUCGGAGAAdTdT-3').

Cell transfection. Cell transfection with plasmids or siRNAs was conducted using Lipofectamine 2000 (Invitrogen), according to the manufacturer's instructions.

Cell proliferation and colony formation assay. For the cell proliferation assay, HCC cells $\left(3-5 \times 10^{3}\right.$ per well) were seeded into 96 -well plates in $100 \mu \mathrm{l}$ culture medium. Cell viability was measured daily at $450 \mathrm{~nm}$ using the Cell Counting Kit-8 (Dojindo Laboratories), according to the manufacturer's instructions. For colony formation, HCC cells $\left(8 \times 10^{4}\right.$ per $10 \mathrm{~cm}$ plate) transfected with empty vector or pcNDA3.1-C9orf100 were selected in an appropriate medium with the addition of $0.8 \mathrm{mg} / \mathrm{ml} \mathrm{G} 418$ (Life Technologies). After 3 weeks, the colonies stained with crystal violet were photographed and counted. All experiments were performed in triplicate.

Western blot analysis. Cell lysates from HCC cells were prepared using cold lysis buffer [25 mmol/1 Tris-Cl (pH 7.5), $1 \%$ SDS, 5 mmol/l EDTA, protease inhibitor cocktail (Sigma, St. Louis, MO, USA)]. Samples were boiled for $5 \mathrm{~min}$, subjected to electrophoresis in SDS-PAGE and transferred onto a polyvinylidene difluoride (PVDF) membrane. The membrane was blocked in 5\% blocking buffer (5\% non-fat milk and $0.1 \%$ Tween-20 in PBS) for $2 \mathrm{~h}$ at room temperature and then incubated with the primary antibody in PBST (0.1\% Tween-20 in PBS) overnight at $4^{\circ} \mathrm{C}$. Incubation with the secondary antibody was performed for $1 \mathrm{~h}$ at room temperature. Anti-Flag (Sigma) and $\beta$-actin (Santa Cruz 

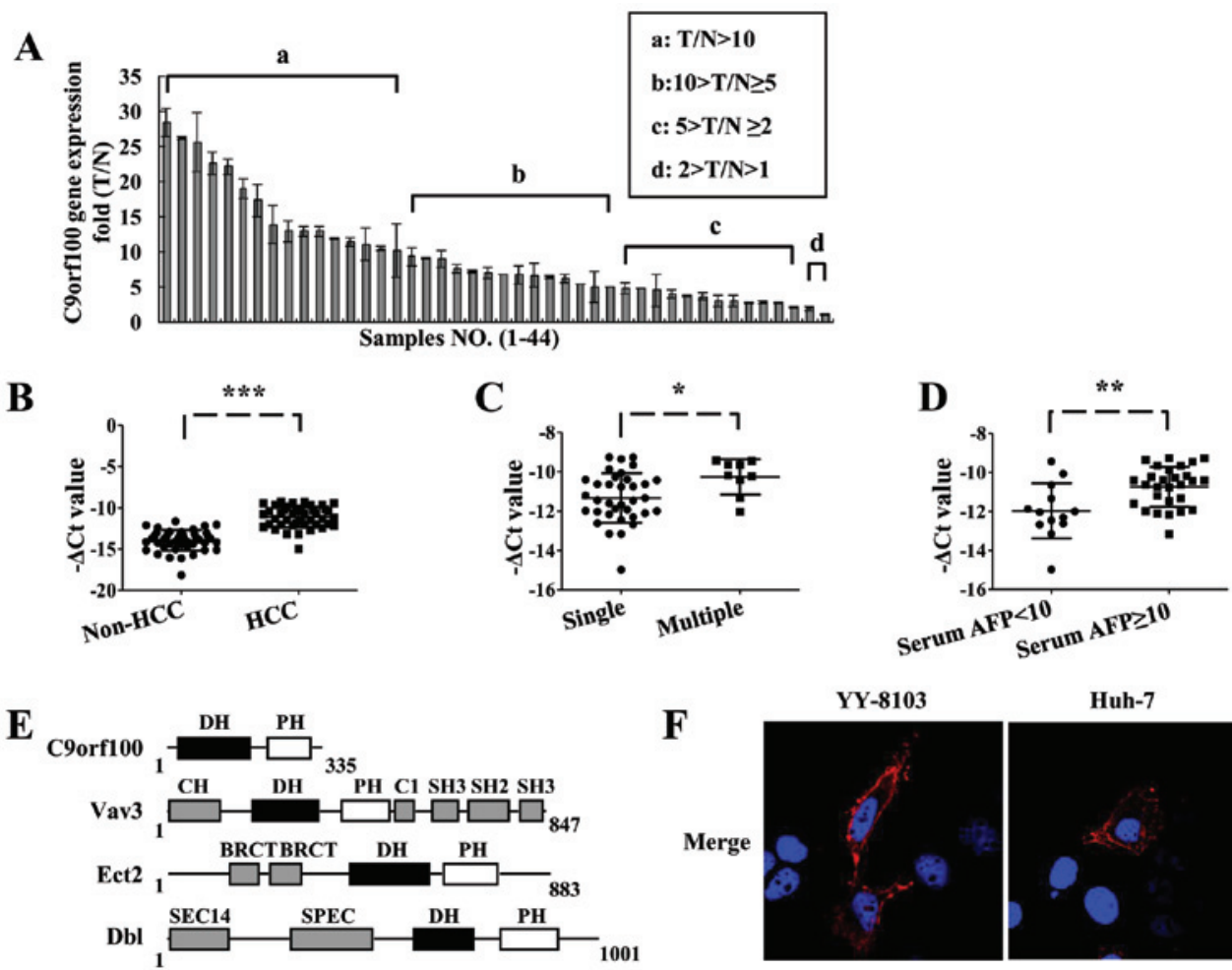

Huh-7

Figure 1. Expression pattern of C9orf100 in HCC specimens. (A) The expression of C9orf100 in 44 pairs of HCC and non-HCC tissues was measured by quantitative real-time PCR. The mean ratio of C9orf100 mRNA levels in HCC tissues to those in non-tumor tissues (T/N) in each case is indicated by a column. (B) C9orf100 mRNA levels were higher in HCC tissues than paired adjacent non-cancerous tissues. (C) C9orf100 mRNA levels were higher in samples with multiple tumors than those with a single tumor. (D) C9orf100 mRNA levels were higher in samples with a serum $\alpha$-fetoprotein level (AFP) of $\geq 10$ ng/ml than those $<10 \mathrm{ng} / \mathrm{ml}$. Relative transcript levels were normalized by the $\Delta \mathrm{Ct}$-method using $\beta$-actin as an internal reference. Medial horizontal lines indicated the mean $-\Delta \mathrm{Ct}$ value of each group. The upper and lower lines are the mean $\pm \mathrm{SD}$. Differences were significant at ${ }^{*} \mathrm{P}<0.05,{ }^{* * *} \mathrm{P}<0.01$ and ${ }^{* * *} \mathrm{P}<0.001$. $(\mathrm{E}) \mathrm{Sch} e \mathrm{matic}$ depiction of the functional domains in C9orf100, Vav3, Ect2 and Dbl. (F) Exogenous C9orf100 protein was localized cell membrane in YY-8103 and Huh-7 cells as indicated in merged images.

Biotechnology, Santa Cruz, Ca, USA) antibodies were used in this study. Detection of proteins was achieved using the Odyssey Infared Imaging System (Li-COR).

Immunofluorescence assay. HCC cells grown on coverslips were washed twice with PBS, fixed with $4 \%$ paraformaldehyde on ice for $30 \mathrm{~min}$ and blocked with $5 \%$ horse serum at room temperature. The cells were then stained with mouse anti-Flag antibody (1:50; Sigma) at $4^{\circ} \mathrm{C}$ overnight, followed by incubation with $\mathrm{Cy} 5$-conjugated anti-mouse secondary antibody (1:200, red; Molecular Probes, Inc.) at room temperature for $30 \mathrm{~min}$. After rinsing 3 times with PBST, the cells were stained for nuclei with 4,6-diamidino-2-phenylindole (DAPI) and analyzed by the Zeiss confocal microscope.

Cell migration assay. Cell migration assays were performed using 24-well transwells (8- $\mu \mathrm{m}$ pore size, BD Biosciences), according to the manufacturer's instructions. Briefly, HCC cells were trypsinized and washed 3 times in DMEM without FBS. A total of $5 \times 10^{4}$ cells was then suspended in $500 \mu \mathrm{l}$ DMEM without FBS and added to the upper chamber, while $750 \mu \mathrm{l}$ DMEM containing 10\% FBS was placed in the lower chamber. The cells were incubated for $24-48 \mathrm{~h}$ at $37^{\circ} \mathrm{C}$ in a $5 \% \mathrm{CO}_{2}$-humidified incubator. The non-migrating cells were removed with cotton swabs and the migrated cells were fixed in $4 \%$ paraformaldehyde and stained with $0.5 \%$ crystal violet. Cells in at least 5 random microscopic fields (magnification, $\mathrm{x} 100$ ) were counted and photographed. All experiments were performed in duplicate and repeated 3 times.

Cell cycle analysis. Flow cytometry was used to analyze the cell cycle. For DNA content analysis, MHCC-97H cells transfected with C9orf100-specific siRNA or control siRNA were harvested at different time-points, fixed in cold $70 \%$ ethanol, washed and rehydrated in PBS. DNA staining was achieved by treating the cells with RNase A $(10 \mathrm{mg} / \mathrm{ml})$ for $30 \mathrm{~min}$ and with propidium iodide $(10 \mu \mathrm{g} / \mathrm{ml}$; Sigma) for $5 \mathrm{~min}$.

Statistical analysis. Statistical analyses were evaluated with Student's t-test using GraphPad Prism 5 software. P>0.05 was considered to indicate a statistically significant difference.

\section{Results}

C9orf100 is frequently upregulated in HCC and is associated with clinicopathologicalfeatures. From a cDNA microarray of paired HCC tissue samples performed in our lab (unpublished data), we found and focused on a gene, C9orf100, which in all cases was significantly upregulated in HCC tissues compared with their non-cancerous counterparts. To confirm this result, 
A

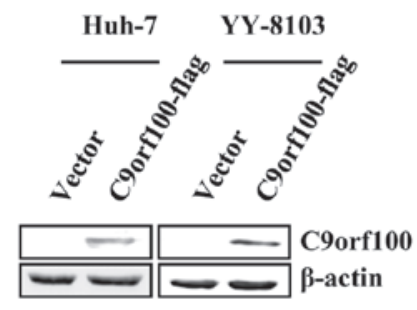

C

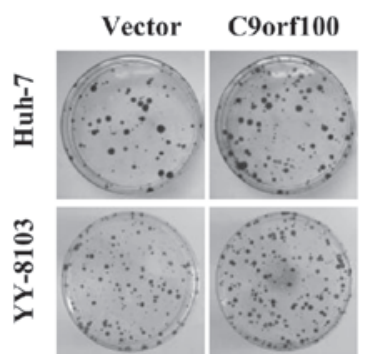

B
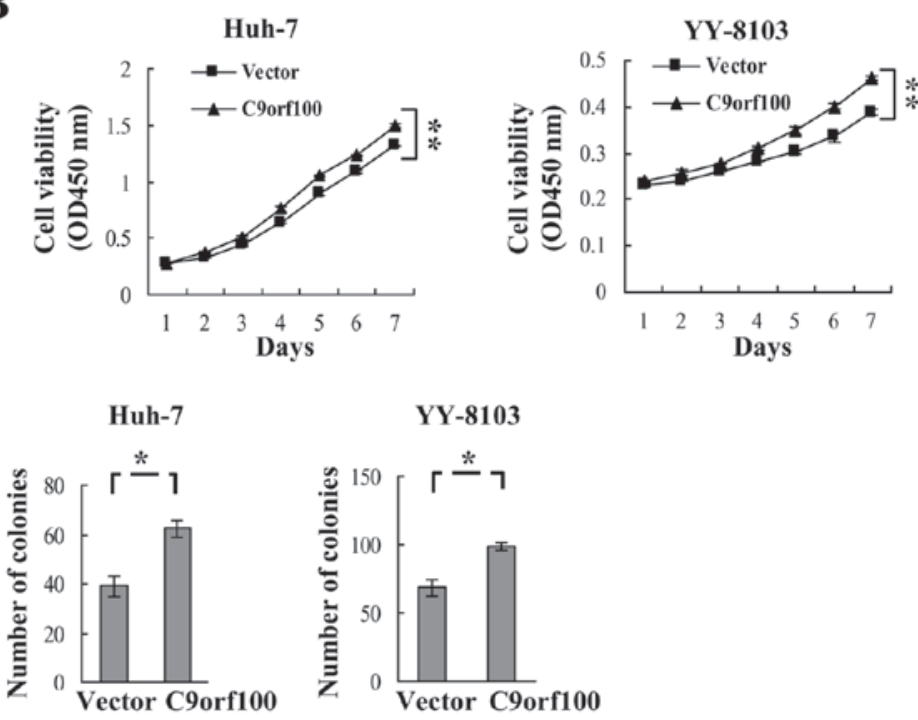

Figure 2. C9orf100 overexpression promoted HCC cell proliferation and colony formation. (A) The ectopic expression of C9orf100 in Huh-7 and YY-8103 cells was analyzed by western blot analysis. The cells transfected with pcDNA3.1 were used as a control. (B) Exogenous C9orf100 expression promoted cell proliferation in Huh-7 and YY-8103 cells. (C) Exogenous C9orf100 expression promoted the colony formation of Huh-7 and YY-8103 cells. The number of colonies was counted and analyzed. The bars are the standard deviation of independent experiments conducted in triplicate $($ mean $\pm \mathrm{SD})$. ${ }^{*}<0.05,{ }^{* *} \mathrm{P}<0.01$.

we detected C9orf100 expression in an additional 44 pairs of HCC tissues by quantitative real-time PCR. In accordance with microarray data, the mRNA level of C9orf100 in HCC samples was markedly higher than that of the corresponding adjacent non-cancerous tissues (Fig. $1 \mathrm{~B}, \mathrm{P}<0.001$ ). Among the 44 pairs of clinical samples, $42 / 44(95.5 \%)$ exhibited at least a 2-fold increase, 30/44 (68.2\%) exhibited a >5-fold increase and none of the cases showed any decrease (Fig. 1A). More notably, C9orf100 mRNA levels were higher in samples with multiple tumors than those with a single tumor (Fig. $1 \mathrm{C}, \mathrm{P}<0.05)$ and with a serum $\alpha$-fetoprotein level (AFP) of $\geq 10 \mathrm{ng} / \mathrm{ml}$ than those $<10 \mathrm{ng} / \mathrm{ml}$ (Fig. 1D, $\mathrm{P}<0.01$ ). However, the upregulation of C9orf100 was not statistically correlated with gender, age, tumor size or other clinicopathological characteristics. Taken together, the expression pattern of C9orf100 in clinical samples suggested it is important in the pathogenesis of HCC.

C9orf100 is a member of the Dbl-family GEFs. Homo sapiens gene $C 9$ orf100 is located on 9p13.3 and encodes a predicted $38-\mathrm{kDa}$ protein of 335 amino acids. The encoded protein belongs to the Dbl-family GEFs, as it shares a DH domain followed by a PH domain. In contrast to other members of this family, C9orf100 lacks additional functional domains in structure (Fig. 1E). We also examined its subcellular distribution of C9orf100 in Huh-7 and YY-8103 cells. As expected, exogenous Flag-tagged C9orf100 was mainly localized to the cell membrane, as indicated by the immunofluorescence assay (Fig. 1F), suggesting it functions as a GEF of Rho GTPases in or near the cell membrane.

Ectopic expression of C9orf100 promotes cell proliferation and colony formation. To determine whether C9orf100 affects HCC cell proliferation, recombinant pcDNA3.1C9orf100 was transfected into Huh-7 and YY-8103 cells.
Results of the western blot analysis showed that C9orf100 was successfully expressed in the two cell lines (Fig. 2A). Cell viability assays using Cell Counting Kit-8 demonstrated that cells transfected with pcDNA3.1-C9orf100 grew more rapidly than those transfected with empty vector in Huh-7 and YY-8103 cells, respectively (Fig. 2B, P<0.01). Moreover, the colony formation assay was also performed to determine the long term effects of C9orf100 on cell growth. Following transfection and selection with G418 for 3 weeks, more colonies were formed in Huh-7 and YY-8103 cells transfected with exogenous $\mathrm{C} 90$ orf100 than the controls (Fig. 2C, $\mathrm{P}<0.05$ ), indicating that $\mathrm{C} 9$ orf 100 overexpression promoted cell proliferation of HCC cells.

Silencing of C9orf100 inhibits cell growth and induces cell cycle delay. To further elucidate the function of C9orf100, we then examined whether a reduction of endogenous C9orf100 expression suppresses cell growth in contrast with promotion by overexpression. To knock down C9orf100 expression, two different small interference RNAs (siRNAs) were chemically synthesized. Quantitative real-time PCR was performed to evaluate their interference efficiency. Compared with the control, si-NC, si-693 and si-1023 strongly decreased the mRNA level of endogenous C9orf100 in MHCC-97H and PLC/PRF/5 cells (Fig. 3A). Cell viability assays demonstrated that effective silencing of $\mathrm{C} 9$ orf 100 significantly inhibited the cell growth of MHCC-97H and PLC/PRF/5 cells (Fig. 3B), suggesting that $\mathrm{C} 9$ orf100 knockdown induced the suppression of cell growth.

To understand the mechanism by which C9orf100 affects cell growth, we performed flow cytometry to detect the cell cycle distribution of MHCC-97H cells at different time-points following transfection. As shown in Fig. 3C, silencing of C9orf100 with si-693 and si-1023 led to a $\mathrm{G}_{2} / \mathrm{M}$ phase arrest at 
A

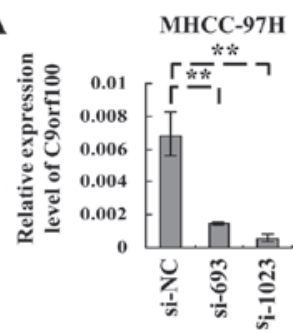

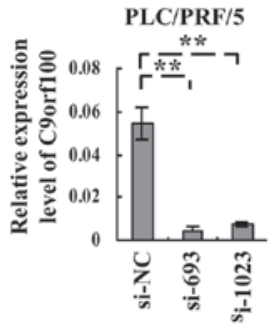

C

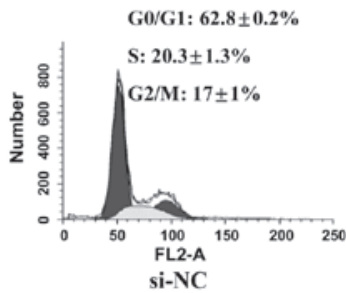

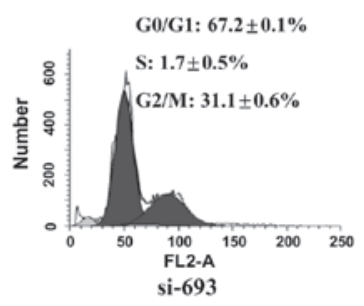

B
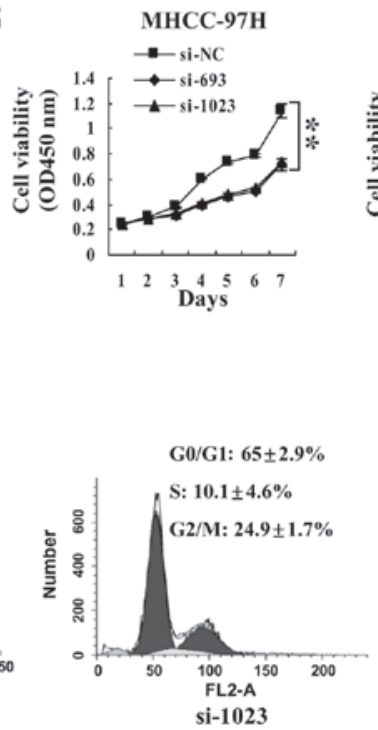
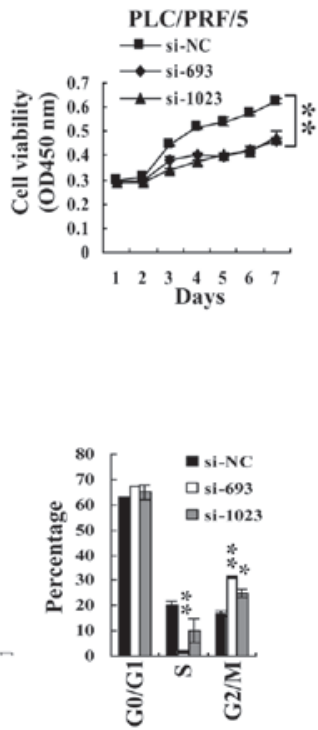

Figure 3. Effect of C9orf100 knockdown on cell growth and cell cycle of HCC cells. (A) Synthesized si-693 and si-1023 knocked down the endogenous C9orf100 in MHCC-97H and PLC/PRF/5 cells, as indicated by quantitative real-time PCR. (B) C9orf100 knockdown suppressed the cell growth of MHCC-97H and $\mathrm{PLC} / \mathrm{PRF} / 5$ cells, where si-NC was used as control. Data were presented as the mean \pm SD of three independent experiments. (C) MHCC-97H cells were harvested at $72 \mathrm{~h}$ following transfection with si-693 or si-1023 and analyzed by flow cytometry. The cells transfected with si-NC were used as a control. The histogram showed the mean and standard deviation of three independent experiments. ${ }^{*} \mathrm{P}<0.05,{ }^{* *} \mathrm{P}<0.01$.

A

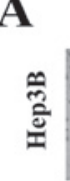

B

Vector
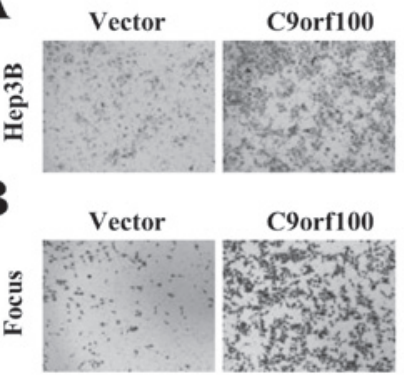

C

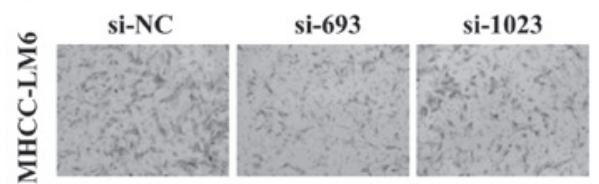

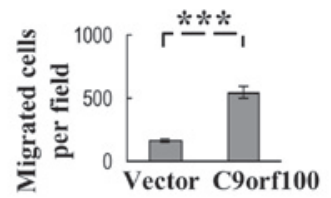
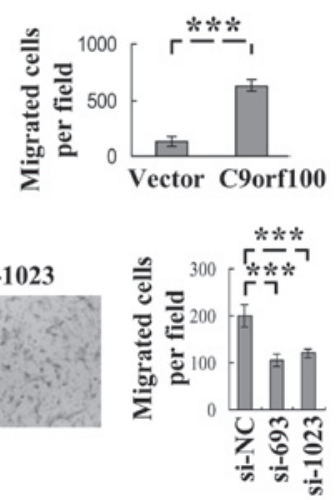

Figure 4. C9orf100 positively regulated HCC cell migration. (A and B) Transwell migration of Hep3B and Focus cells were performed after transfection with pcNDA3.1-C9orf100 or empty vector. (C) Transwell migration assay for MHCC-LM6 cells. The cells transfected with si-NC were used as control. The histogram showed the calculated average number of migrated cells per field (mean $\pm \mathrm{SD}, \mathrm{n}=5$ ). Statistical analysis was performed with Student's t-test; ${ }^{* * *} \mathrm{P}<0.001$.

$72 \mathrm{~h}$. The cells transfected with si-NC were used as the control. This result suggested that $\mathrm{C} 9$ orf100 was involved in the cell cycle control of the $\mathrm{G}_{2} / \mathrm{M}$ phase.

C9orf100 is involved in the positive regulation of $\mathrm{HCC}$ cell migration. Intrahepatic cancer metastasis is a critical aspect of HCC recurrence following hepatectomy. We have demonstrated that C9orf100 expression was significantly higher in the group with multiple tumors than in the group with a single tumor in clinical samples, suggesting it plays a role in intrahepatic metastasis. To verify this hypothesis, we employed a transwell chamber assay to access the effect of C9orf100 on cell migration. After suspending the cells in the upper chamber for $24 \mathrm{~h}$, we found that Hep3B cells transiently transfected with pcDNA3.1-C9orf100 migrated to the lower chamber more easily than those transiently transfected with the empty vector. There was a 3 -fold increase in Hep3B/ C9orf100 compared to Hep3B/vector cells (Fig. 4A, P<0.001). A similar result was also observed in Focus cells (Fig. 4B, $\mathrm{P}<0.001$ ). Of note, the ectopic expression of C9orf100 in Hep3B and Focus cells had no impact on cell proliferation within $48 \mathrm{~h}$ (data not shown), suggesting that the increased migration rate induced by C9orf100 was independent of cell proliferation. By contrast, when C9orf100 was knocked down by specific siRNAs in MHCC-LM6 cells, the migrated cells from the upper chamber were obviously reduced relative to those treated with si-NC (Fig. 4C, P<0.001). Taken together, these data demonstrated that an increase or decrease in the C9orf100 level had a strong impact on cell migration, indicating that C9orf100 may contribute to the metastasis of HCC.

\section{Discussion}

C9orf100 is a new gene and its identification has not previously been reported. Little is known about C9orf100 except that it is supposed to be a member of the Dbl-Family GEFs in structure. In the present study, we provide compelling evidence, for the first time, that C9orf100 serves as a potential oncogene in HCC. C9orf100 overexpression was found in $95.5 \%$ of HCC samples used in our investigation and was correlated with HCC clinicopathological features. Furthermore, the enhanced expression of C9orf100 promoted cell proliferation and the 
knockdown of endogenous C9orf100 suppressed cell growth by inducing cell cycle arrest in the $\mathrm{G}_{2} / \mathrm{M}$ phase. Additionally, we demonstrated that C9orf100 was involved in the positive regulation of $\mathrm{HCC}$ cell migration. These data strongly suggest that C9orf100 mediates the development and progression of HCC.

To date, only a few biomarkers have been applied to diagnose HCC. $\alpha$-fetoprotein (AFP), for its best specificity and sensitivity, has been extensively used in clinics; however it remains insufficient for identifying $\mathrm{HCC}$ at the early stage of tumor formation (20). Furthermore, tumor multiplicity is an adverse prognostic factor in the AJCC staging systems. Patients with multinodular HCCs have a poorer 5-year diseasefree outcome and overall survival rate than those with large solitary tumors who have undergone liver resection (21). Given that C9orf100 was highly and widely upregulated in the HCC samples, particularly closely associated with the AFP level and tumor multiplicity (Fig. 1C and D), it is likely that C9orf100 may be applied as a novel biomarker for HCC early diagnosis or prognosis outcome. Nevertheless, further experiments still need to be conducted.

Sequence analysis of C9orf100 predicted it possesses a $\mathrm{DH}-\mathrm{PH}$ module, which is commonly shared in the Dbl-family GEFs. We have shown the difference between C9orf100 and other known members of this family (Fig. 1E). Despite lacking additional functional domains, C9orf100 still exhibited a marked oncogenic role as other members do, suggesting the minimal catalytic unit of the Dbl-family GEFs is sufficient to maintain the active GTP-bound states of Rho GTPases. RhoA, Rac1, and Cdc42 are the main members of Rho GTPases that have previously been reported as key regulators of cell migration (12). GEFs such as Dbs and Dbl are able to activate both Cdc42 and RhoA (22), whereas Vav3 catalyzes exchange only on RhoA and Racl (23), indicating GEFs have their substrate specificity. Further research is required to determine which Rho GTPase serves as the substrate of C9orf100. Moreover, the mechanism by which C9orf100 promotes cell proliferation and migration via Rho GTPases should also be explored in the future.

In summary, we identified C9orf100, the new member of the Dbl-family GEFs, as a candidate oncogene in HCC. Our findings may provide new insight into identifying a novel diagnostic biomarker and developing a new therapeutic strategy against HCC. Furthermore, our study has also allowed us to gain a better understanding of the Dbl-family GEFs in tumor development.

\section{Acknowledgements}

We thank our laboratory members, Qing Deng, Ruifang Liu, $\mathrm{Xiao} \mathrm{Xu}$ and Fei Chen for their helpful discussion and generous technical assistance. This study was supported by grants from the Academician Workstation of Wuxi People's Hospital (CYE00917), the Chinese National Key Program on Basic Research (2010CB529204) and the China National Key Projects for Infectious Disease (2008ZX10002-021).

\section{References}

1. Schafer DF and Sorrell MF: Hepatocellular carcinoma. Lancet 353: 1253-1257, 1999.

2. Thorgeirsson SS and Grisham JW: Molecular pathogenesis of human hepatocellular carcinoma. Nat Genet 31: 339-346, 2002.

3. Llovet JM, Burroughs A and Bruix J: Hepatocellular carcinoma. Lancet 362: 1907-1917, 2003.

4. Bruix J, Hessheimer AJ, Forner A, Boix L, Vilana R and Llovet JM: New aspects of diagnosis and therapy of hepatocellular carcinoma. Oncogene 25: 3848-3856, 2006.

5. Iizuka N, Oka M, Yamada-Okabe H, et al: Comparison of gene expression profiles between hepatitis B virus- and hepatitis C virus-infected hepatocellular carcinoma by oligonucleotide microarray data on the basis of a supervised learning method. Cancer Res 62: 3939-3944, 2002.

6. Oliva J, Bardag-Gorce F, French BA, et al: Fat10 is an epigenetic marker for liver preneoplasia in a drug-primed mouse model of tumorigenesis. Exp Mol Pathol 84: 102-112, 2008.

7. Huang J, Zheng DL, Qin FS, et al: Genetic and epigenetic silencing of SCARA5 may contribute to human hepatocellular carcinoma by activating FAK signaling. J Clin Invest 120: 223-241, 2010

8. Huang J, Zhang X, Zhang M, et al: Up-regulation of DLK1 as an imprinted gene could contribute to human hepatocellular carcinoma. Carcinogenesis 28: 1094-1103, 2007.

9. Okada T, Iizuka N, Yamada-Okabe H, et al: Gene expression profile linked to $\mathrm{p} 53$ status in hepatitis $\mathrm{C}$ virus-related hepatocellular carcinoma. FEBS Lett 555: 583-590, 2003.

10. Wettschureck $\mathrm{N}$ and Offermanns S: Rho/Rho-kinase mediated signaling in physiology and pathophysiology. J Mol Med (Berl) 80: 629-638, 2002.

11. Etienne-Manneville S and Hall A: Rho GTPases in cell biology. Nature 420: 629-635, 2002.

12. Raftopoulou M and Hall A: Cell migration: Rho GTPases lead the way. Dev Biol 265: 23-32, 2004.

13. Hart MJ, Eva A, Zangrilli D, et al: Cellular transformation and guanine nucleotide exchange activity are catalyzed by a common domain on the dbl oncogene product. J Biol Chem 269: 62-65, 1994.

14. Baumeister MA, Rossman KL, Sondek J and Lemmon MA: The Dbs PH domain contributes independently to membrane targeting and regulation of guanine nucleotide-exchange activity. Biochem J 400: 563-572, 2006.

15. DiNitto JP and Lambright DG: Membrane and juxtamembrane targeting by PH and PTB domains. Biochim Biophys Acta 1761: 850-867, 2006.

16. Rossman KL, Der CJ and Sondek J: GEF means go: turning on RHO GTPases with guanine nucleotide-exchange factors. Nat Rev Mol Cell Biol 6: 167-180, 2005.

17. Schmidt A and Hall A: Guanine nucleotide exchange factors for Rho GTPases: turning on the switch. Genes Dev 16: 1587-1609, 2002.

18. Eva A, Vecchio G, Rao CD, Tronick SR and Aaronson SA: The predicted DBL oncogene product defines a distinct class of transforming proteins. Proc Natl Acad Sci USA 85: 2061-2065, 1988.

19. Salhia B, Tran NL, Chan A, et al: The guanine nucleotide exchange factors trio, Ect2, and Vav3 mediate the invasive behavior of glioblastoma. Am J Pathol 173: 1828-1838, 2008.

20. Pang RW, Joh JW, Johnson PJ, Monden M, Pawlik TM and Poon RT: Biology of hepatocellular carcinoma. Ann Surg Oncol 15: 962-971, 2008.

21. Ramacciato G, Mercantini P, Petrucciani N, et al: Does surgical resection have a role in the treatment of large or multinodular hepatocellular carcinoma? Am Surg 76: 1189-1197, 2010.

22. Whitehead IP, Lambert QT, Glaven JA, et al: Dependence of Dbl and Dbs transformation on MEK and NF- $\mathrm{\kappa B}$ activation. Mol Cell Biol 19: 7759-7770, 1999.

23. Movilla N and Bustelo XR: Biological and regulatory properties of Vav-3, a new member of the Vav family of oncoproteins. Mol Cell Biol 19: 7870-7885, 1999. 